\title{
A pilot investigation of audiovisual processing and multisensory integration in patients with inherited retinal dystrophies
}

\author{
Mark H. Myers ${ }^{1 *}$ D, Alessandro lannaccone ${ }^{2}$ and Gavin M. Bidelman ${ }^{1,3,4}$
}

\begin{abstract}
Background: In this study, we examined audiovisual (AV) processing in normal and visually impaired individuals who exhibit partial loss of vision due to inherited retinal dystrophies (IRDs).

Methods: Two groups were analyzed for this pilot study: Group 1 was composed of IRD participants: two with autosomal dominant retinitis pigmentosa (RP), two with autosomal recessive cone-rod dystrophy (CORD), and two with the related complex disorder, Bardet-Biedl syndrome (BBS); Group 2 was composed of 15 non-IRD participants (controls). Audiovisual looming and receding stimuli (conveying perceptual motion) were used to assess the cortical processing and integration of unimodal ( $\mathrm{A}$ or $\mathrm{V}$ ) and multimodal (AV) sensory cues. Electroencephalography (EEG) was used to simultaneously resolve the temporal and spatial characteristics of AV processing and assess differences in neural responses between groups. Measurement of AV integration was accomplished via quantification of the EEG's spectral power and event-related brain potentials (ERPs).

Results: Results show that IRD individuals exhibit reduced AV integration for concurrent audio and visual (AV) stimuli but increased brain activity during the unimodal A (but not $V$ ) presentation. This was corroborated in behavioral responses, where IRD patients showed slower and less accurate judgments of $A V$ and $V$ stimuli but more accurate responses in the A-alone condition.
\end{abstract}

Conclusions: Collectively, our findings imply a neural compensation from auditory sensory brain areas due to visual deprivation.

Keywords: Power spectral density analysis, Electroencephalography, Inherited retinal dystrophies, Event-related brain potentials

\section{Background}

Investigation of multi-modal stimulation has found that when auditory (A) and visual (V) stimuli are presented simultaneously, congruent information can change an individual's object perception. If the dual stimulation is staggered in such a way as to convey a delayed time to contact of approaching stimuli, visual stimulation usually provides a more salient response. The timing of multisensory input may thus alter sensory integration in determining behavior and shaping perception [8]. For instance, two stimuli (e.g., concurrent AV stimuli)

\footnotetext{
* Correspondence: mmyers25@uthsc.edu

${ }^{1}$ Department of Anatomy and Neurobiology, University of Tennessee Health Sciences Center, Memphis, TN 38163, USA

Full list of author information is available at the end of the article
}

initiated to disparate sensory areas can result in a facilitation of enhanced sensory-perceptual processing [18].

Human neuroimaging studies reveal several underlying brain mechanisms responsible for AV processing [5]. AV stimuli elicit a complex cortical network featuring activation in the primary auditory and visual cortices, as well as several multisensory areas (superior temporal sulcus, intraparietal sulcus, insula, and pre-central cortex). However, several other studies have also found that unimodal sensory input can influence neural responses found in other distal areas normally responsible for processing different sensory modalities [30]. As an example, lip-reading from visual-only videos of mouth movement is associated with responses in auditory cortices (i.e., Heschl's gyrus and Planum temporale) even if no auditory 
input is available $[6,24,37]$. These studies illustrate a dynamic interplay and cross-talk between uni- and multimodal brain areas during AV processing.

The investigation of looming (approaching) and receding (fading) signals is a particularly promising avenue to address synergy between principles of multisensory processing. Looming signals dynamically increase in their effectiveness and spatial coverage relative to receding stimuli. It is also noteworthy that looming cues can indicate both potential threats/collisions and success in acquiring sought-after objects/goals [19, 21, 43], suggesting they are of high behavioral relevance.

In this study, we focused on AV integration in cases of inherited retinal dystrophies (IRDs), in order to further understand the neural mechanisms of multi-modal processing. IRD is a group of degenerative retinal diseases causing a progressive loss of photoreceptor cells. While the retinal micro-anatomical and functional visual characteristics of IRD have been and remain the object of intense investigation, its perceptual-cognitive consequences on AV processing remain largely unknown (cf. $[1,20])$.

Several pieces of evidence suggest that IRDs might alter multisensory processing. Interestingly, IRD individuals often report that other sensory capabilities (e.g., hearing, taste) become more acute over time $[1,20]$. The sensitization or marshalling of additional sensory areas has been known to compensate for singular sensory loss. For example, in individuals with early onset blindness, loud sounds can induce the illusory perception of flashes of light, suggesting an increase in visual awareness for certain auditory stimuli [1]. Such occurrences of putative 'remapping' or sensory 'cross-talk' may be the result of de-afferentation where sensory input to the auditory system begins innervating (or establishes stronger connections) to extra-striate visual areas. These cases reinforce the notion that connections in the adult brain can be modified when one sensory system becomes deficient and others are forced to provide compensatory processing.

In the current study, we aimed to characterize how the unimodal visual deficits of IRDs alter the perception and neurophysiological processing of AV stimuli, when the brain must bind (i.e., integrate) sound and visual cues. In this regard, IRD patients offer an ideal window for examining how visual deprivation might change sensory processing in a different modality (e.g., auditory) as well in tandem with the impaired visual sensory input (i.e., during AV processing). We show that IRD individuals have reduced AV processing and/or integration of multisensory cues, consistent with their impaired unimodal visual input, and those IRD patients demonstrate increased responsiveness to auditory stimuli, consistent with the notion that visual deficits are partially compensated by recruitment and perhaps expansion of auditory function [17]. We also assessed response identification accuracy (\%) and reaction times (RTs) of the participants during AV processing to understand the behavioral implications of IRD's putative cortical remapping on perception and motor control.

\section{Methods}

Six patients (5 males, 1 female; mean $\pm S D$ age $=46.7 \pm$ $23 \mathrm{yrs}$ ) with different types of IRDs were recruited in this pilot study: two with autosomal dominant (AD) retinitis pigmentosa (RP), two with autosomal recessive (AR) cone-rod dystrophy (CORD), and two with the related complex disorder, Bardet-Biedl syndrome (BBS) $[15,25-28]$. Fifteen healthy non-IRD control participants (13 females, 2 males; age $=23.6 \pm 2$ yrs) were also recruited as baseline controls for this study. All participants had normal hearing by self-report. This study had the joint approval of the Institutional Review Boards of the University of Tennessee Health Science Center (IRB \#: 13-02782-XP) and the University of Memphis (IRB \#2370).

Diagnoses of the IRD patients was established via a combination of physician evaluation by a retinal degeneration expert (AI), psychophysical (Goldmann visual fields; dark- and light-adapted monochromatic automated perimetry), dark- and light-adapted flash electroretinogram (ERG), and imaging [spectral domain optical coherence tomography (SD-OCT) and fundus autofluorescence (FAF)] methods, and were further confirmed whenever possible (in 5 of the 6 participants) via molecular genetic diagnostic testing. The characteristics of the six patients are summarized in Table 1.

IRD patients were selected for this study to be representative of three different scenarios (Additional file 1: Figure S1): (i) severe peripheral vision loss with concentric visual field constriction and central visual preservation as in classical RP, in which the primary cells affected by the disease are the rods ( $\operatorname{rod}>$ cone disease; Additional file 1: Figure S1A-B); (ii) severe central vision loss with peripheral preservation as in CORD, in which there is a cone $>$ rod disease pattern (Additional file 1: Figure S1C-D); and (iii) moderately severe vision loss across the board with moderate to fair preservation of both peripheral and central vision as in BBS patients, a ciliopathy in which both rods and cones are affected with marked shortening of the outer segment but, at least at the initial stages we chose, with only ring scotomas and fairly good preservation of both peripheral and central visual function (Additional file 1: Figure S1E-F). The objective was that of choosing patients representative of vision loss patterns to begin testing the hypothesis that all scenarios would affect the response to A, V and AV stimuli, but that they would differ in their abnormality patterns to looming vs. receding stimuli, with BBS patients predicted to be the closest to normal to both types of stimuli. 


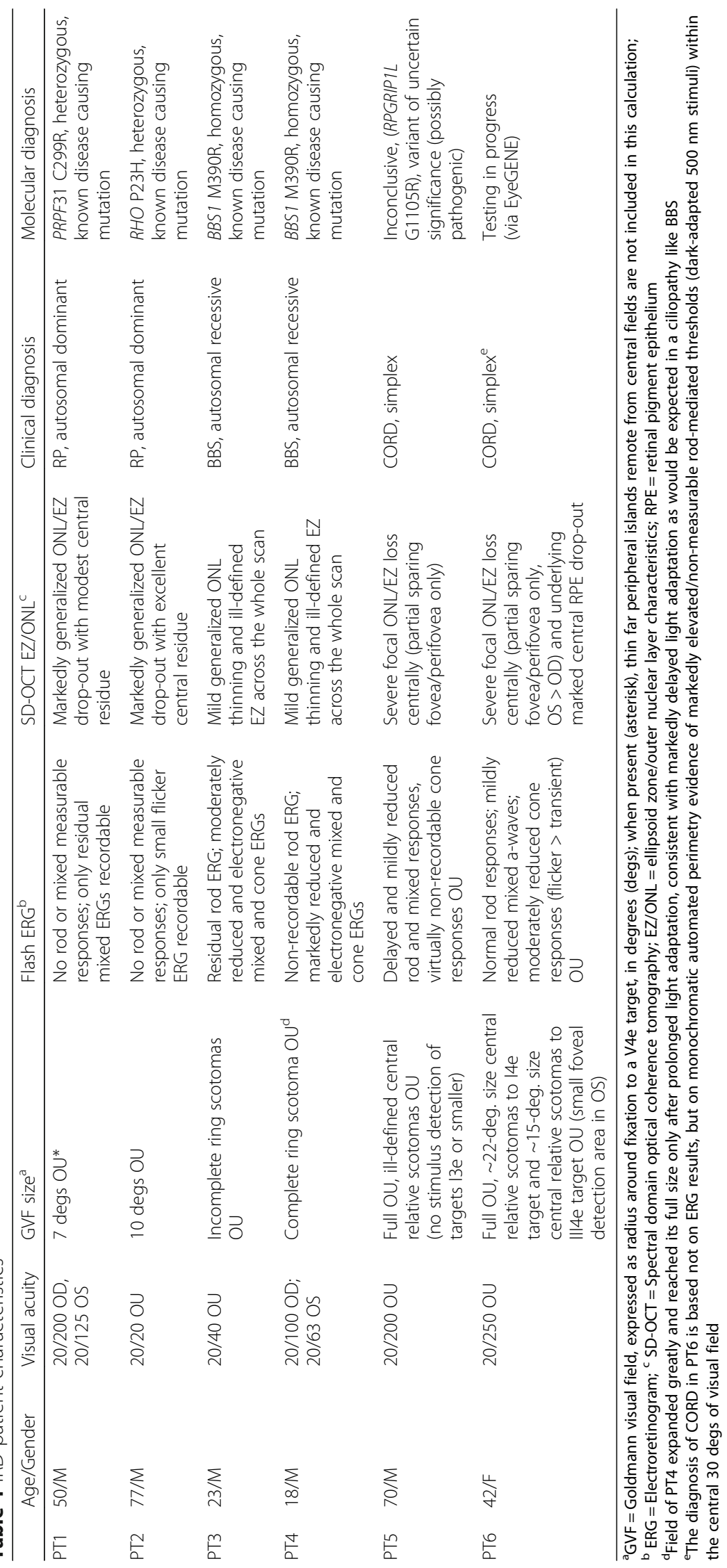




\section{Audiovisual stimuli and behavioral task}

The experiment involved the identification of stimuli that could be looming or receding auditory, visual, or multisensory audiovisual ( $\mathrm{A}, \mathrm{V}$, and $\mathrm{AV}$, respectively), as described in Cappe et al. [14]. To induce the perception of looming movement, visual stimuli (detailed below) changed in size and auditory stimuli changed in intensity by $80 \mathrm{~dB}$ over $1000 \mathrm{~ms}$ so as to give the impression of "looming" (i.e., approaching; $0 \rightarrow 80 \mathrm{~dB}$ SPL) or "receding" $(80 \rightarrow 0 \mathrm{~dB}$ SPL) movement. In the present study, looming stimuli were treated as targets and receding stimuli as catch trials (not analyzed in this study). Each the three conditions were repeated 150 times across 15 blocks of randomly intermixed trials. On each trial, participants were asked to judge if the stimulus was "looming" or "receding" via a button press on the computer. They were encouraged to respond as accurately and as quickly as possible. Both response identification (\%) and reaction times (RTs) were recorded. The stimulus conditions are schematized in Fig. 1.

\section{Auditory stimuli}

Sound tokens comprised $80 \mathrm{~dB}$ rising-intensity (looming signal) and falling intensity (receding signal) $1000 \mathrm{~Hz}$ complex tones composed of a triangular waveform. Auditory stimuli were generated with Adobe Audition software (Adobe Systems Inc.). Prior research has shown that these types of complex tonal stimuli produce more reliable looming and receding percepts [36] and may also be preferentially involved in multisensory integration compared to simple tones [32, 41]. Auditory stimuli were presented over Etymotic ER-2 insert earphones
(Etymotic Research). Tokens were sampled at $48 \mathrm{kHz}$ and were $1000 \mathrm{~ms}$ including $10 \mathrm{~ms}$ onset/offset ramps (to avoid audible clicks).

\section{Visual stimuli}

Visual tokens consisted of a centrally presented disc (white on a black background) that symmetrically expanded (from $7^{\circ}$ to $13^{\circ}$ diameter with the radius increasing linearly at a constant rate) in the case of looming stimuli or contracted (from $13^{\circ}$ to $7^{\circ}$ diameter) in the case of receding tokens. Visual tokens were presented on Dell 27" U2713 LED computer monitor at a distance of $90 \mathrm{~cm}$.

Multisensory AV stimuli featured the combined auditory and visual tokens described above. All stimuli were matched in total duration $(1000 \mathrm{~ms})$. The interstimulus interval was varied from 800 to $1400 \mathrm{~ms}$ (rectangular distribution) to avoid participants anticipating the timing of stimulus presentation. A focal point in the form of a fixation cross $(+)$ was presented on screen between trials to control gaze and minimize saccades. Every participant was able to visualize and fixate foveally the fixation target. Stimulus delivery and response recording was controlled by custom routines coded in MATLAB $^{\circ} 2013$ (The MathWorks, Inc).

\section{EEG recordings}

Evoked brain responses were recorded using Neuroscan SynAmps RT amplifiers. A 64-channel sintered $\mathrm{Ag} / \mathrm{AgCl}$ electrode array (QuikCap, Compumedics NeuroScan) was used to record neuroelectric activity from around the scalp using an average referenced montage. Additional electrodes placed on the outer canthi of the eyes
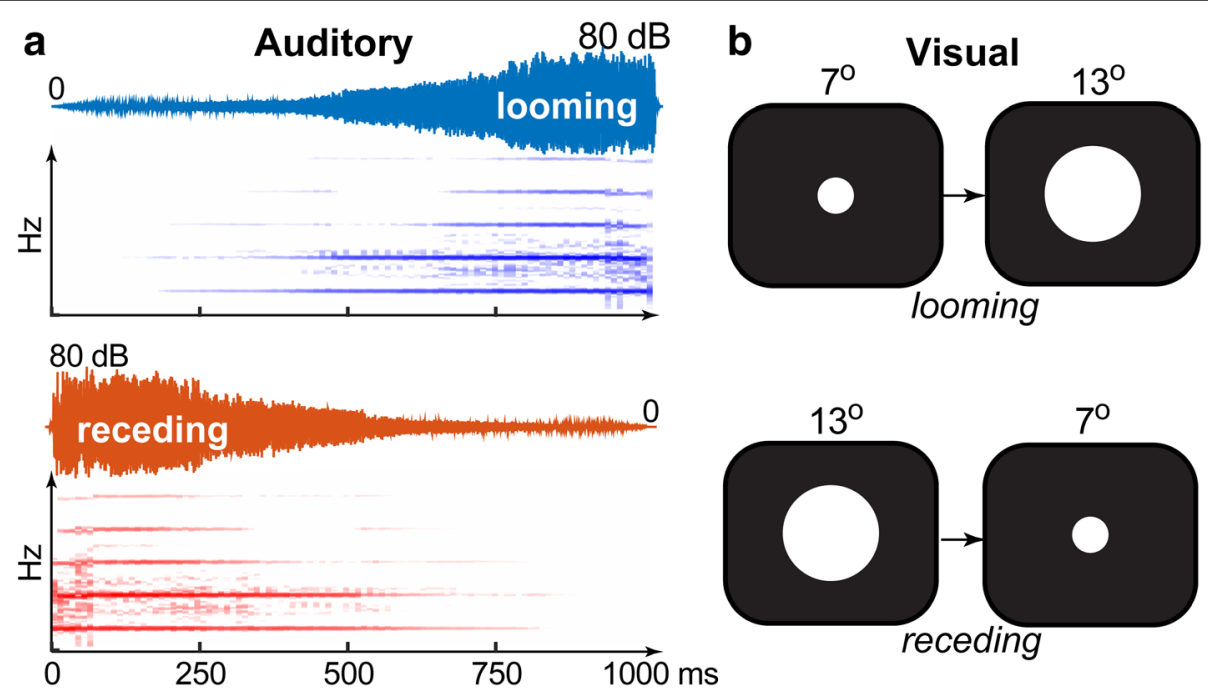

Fig. 1 Stimuli and behavioral paradigm. Participants perform detection of moving (looming, receding) stimuli that could be a auditory, $\mathbf{b}$ visual, or multisensory auditory-visual. a Auditory time waveforms (top) and spectrograms (bottom). b Visual stimuli increased (looming) or decreased (receding) in size. Perception of movement was induced by linearly changing the size of the centrally displayed disk for the visual condition and by changing the intensity of a complex tone for the auditory condition over 1000 ms (e.g., [14]) 
and the superior and inferior orbit monitored ocular activity. Specific EEG hardware and recording parameters (presentation rate, filtering, sample rate, etc.) followed typical procedures from our laboratory [11, 12]. Electrode contact impedance was maintained $<5 \mathrm{k} \Omega$. Subjects reclined comfortably in an electroacoustically shielded IAC sound booth during testing. EEG signals were amplified, online filtered $(0.1-500 \mathrm{~Hz})$, and digitized at $1 \mathrm{kHz}$ per channel. Analysis epoch windows for the cortical event related potentials (ERPs) spanned -100 to $1100 \mathrm{~ms}$ to encompass an appropriate prestimulus interval for baseline correction and the extent of the stimulus trial. Individual epochs contaminated by myogenic noise were manually discarded and blink artifacts $( \pm 50 \mu \mathrm{V})$ rejected prior to averaging using principal component (PCA) decomposition [46]. Preprocessing was conducted in the Curry 7 Neuroimaging Suite (Compumedics Neuroscan) and custom routines coded in MATLAB and EEGLAB [16].

\section{Power spectral density analysis}

Power spectral density (PSD) analysis enables the characterization of neural activity with respect to its frequency distribution. PSDs were computed at each electrode location per subject and stimulus to quantify the amount of neural power (measured in $\mu \mathrm{V}$ ) occurring at a given sensor location and across frequency. An example of the power spectral density measured at a representative electrode is shown in Fig. 2.

Linear regression was then performed across the frequency range in order to quantify the overall tilt of the PSD spectrum over the bandwidth from 1 to $100 \mathrm{~Hz}$. A negative slope value $(-\alpha)$ typically conforms to the fractal dimensionality of $1 / \mathrm{f}^{\alpha}$ (Fig. 2). This analysis enabled us to determine if there was a uniform distribution of power across the scalp EEG (slope $=0$ ), or rather, an increase/decrease in lower- (alpha: 8-15 Hz) vs. higherfrequency (gamma: 20-80 Hz) bands of the EEG (slope $\neq 0$ ). In particular, beta-gamma neural frequency activity has been associated with cognitive processing $[7,9,10]$ and may be associated with congenital neuropathic conditions or trauma to areas of the cortex [33, 34]. PSD slopes were computed for each electrode location and participant. This resulted in a topographic map of PSD values across the scalp. We compared groups' PSDs for each stimulus condition using a $t$-test on the topographic maps (threshold masked at $p<0.05$ ). This approach identified regions of electrode clusters which distinguished AV responses in control and IRD listeners. Multiple comparisons were corrected via the BenjaminiHochberg method to reduce the false discovery rate (FDR) [4].

\section{Statistical analysis}

Two-way mixed model ANOVAs were used to analyze the behavioral data (\%, identification and RTs) with factors group (IRD, control) and stimulus (A, V, AV). Subjects served as a random factor. The behavioral responses we aimed to measure were categorical binary judgments (i.e., "looming" vs. "receding"). Consequently, we used a binomial distribution and canonical logit link function in the ANOVA model for identification scores. RTs were analyzed using a similar ANOVA only with a normal Gaussian distribution to model the residuals, given the continuous nature of RT responses. Although age was not correlated with EEG measures $\left(r_{s}=-0.18\right.$,

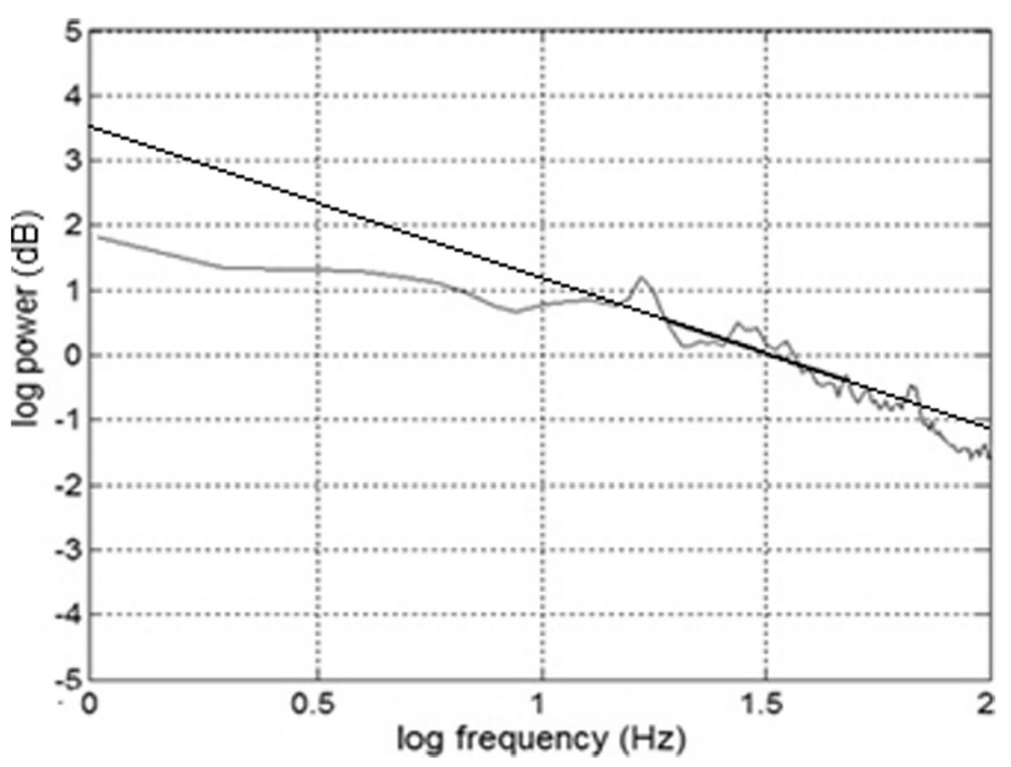

Fig. 2 Linear regression analysis of PSD spectral slopes of the EEG spectrum of control participant 
$p=0.15)$, on average, IRD patients were older than controls (IRD: $46 \pm 23$ yrs.; controls: $23.6 \pm 2$ yrs). Consequently, we used age as covariate in the ANOVA models to partial out potential age-related changes in the evoked potentials ([13]a). An a priori significance level of $a=0.05$ was used for all statistical testing.

\section{Results}

Behavioral identification and reaction times for correctly judging "looming" stimuli are shown in Fig. 3. An ANOVA (logistics model) conducted on identification judgments revealed a significant group $\mathrm{x}$ stimulus interaction $\left[F_{2,38}=86.32, p<0.0001, \eta_{p}^{2}=0.82\right]$, after accounting for age. Multiple comparisons indicated this interaction was attributable to group differences in the $\mathrm{V}(p=0.0248)$ and $\mathrm{AV}(p=0.027)$ conditions; no difference between groups was observed in the unimodal A condition $(p=0.93)$. That is, the propensity to correctly identify stimuli was better in the control relative to IRD group for $\mathrm{V}$ and $\mathrm{AV}$ tokens whereas group identification was similar for A tokens.

Analysis of the RTs similarly revealed a group $\mathrm{x}$ stimulus interaction $\left[F_{2,38}=3.52, p=0.039, \eta_{p}^{2}=0.22\right]$, after accounting for age. Post hoc comparisons revealed that controls were $\sim 400 \mathrm{~ms}$ faster $(1407 \pm 93 \mathrm{~ms})$ in judging audiovisual stimuli than IRD participants (1796 \pm $269 \mathrm{~ms}$ ) across the board [main effect of group: $F_{1,38}=$ 27.71, $\left.p<0.0001 \eta_{p}^{2}=0.42\right]$. Yet, the interaction again suggests this group difference depended on the stimulus condition. Post hoc comparisons indicated that IRD patients were slower at responding than controls for each stimulus conditions (A, V, AV; all $p$-values $<0.0001$ ).
Within the IRD group, patients showed slower RTs when judging unimodal V stimuli compared to multimodal AV stimuli $(p=0.006)$, consistent with their visual deficits. The comparison between RTs for the V and A stimuli in the IRD group was marginal $(p=0.07)$. In contrast, RTs were similar across stimuli for the control group ( $p s>0.25)$, yet trending in the expected direction (fastest for the AV multimodal condition). Collectively, these analyses indicate group differences in the perceptual identification of both $\mathrm{A}$ and $\mathrm{V}$ stimuli that convey motion, with slower and less accurate judgments in IRD patients.

Our initial analysis of possible group differences in multisensory neural encoding assessed time-locked ERP responses to $\mathrm{A}, \mathrm{V}$, and $\mathrm{AV}$ stimuli. ERPs to looming $\mathrm{A}$, $\mathrm{V}$, and AV stimuli are shown for control and IRD patients in Fig. 4. Generally speaking, IRD patients exhibited larger (i.e., more robust) negative $\mathrm{N} 1$ responses (see deflection at $100 \mathrm{~ms}$ ) for A and AV stimulation, suggesting higher sensitivity to auditory stimuli.

To more directly quantify group differences in multisensory neural processing, we computed a difference waveform analysis between the combined AV and unimodal $\mathrm{A}$ and $\mathrm{V}$ conditions [i.e., $\mathrm{AV}-(\mathrm{A}+\mathrm{V})$ ]. This difference potential allowed us to examine the degree to which the multimodal AV stimulus produced a facilitation effect compared to the sum of the unimodal conditions alone [45]. Results of this analysis are shown in Fig. 4c. We used a sample-by-sample $t$-test contrasting these potentials against a zero baseline to assess significance of AV responses per group [10, 23]. That is, the combined AV ERP must be larger than the sum of the unimodal responses to confirm multisensory processing
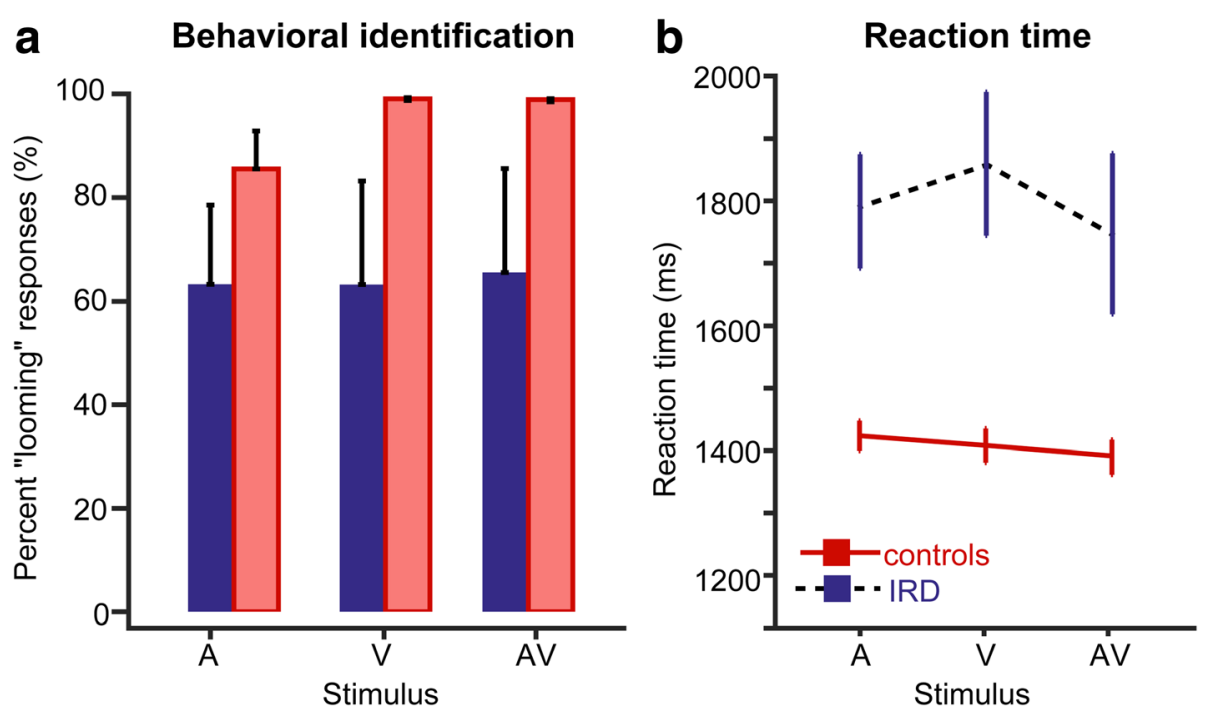

Fig. 3 Grand average behavioral identification (a) and reaction times (b) for correctly judging "looming" audiovisual stimuli for the control and IRD group. Error bars $= \pm 1$ s.e.m 


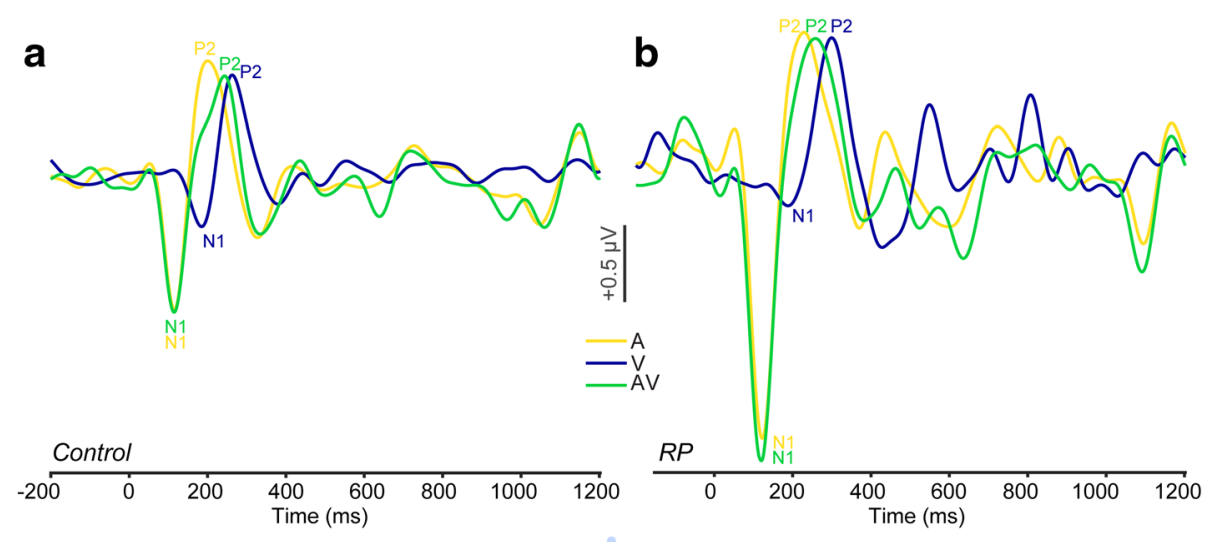

C

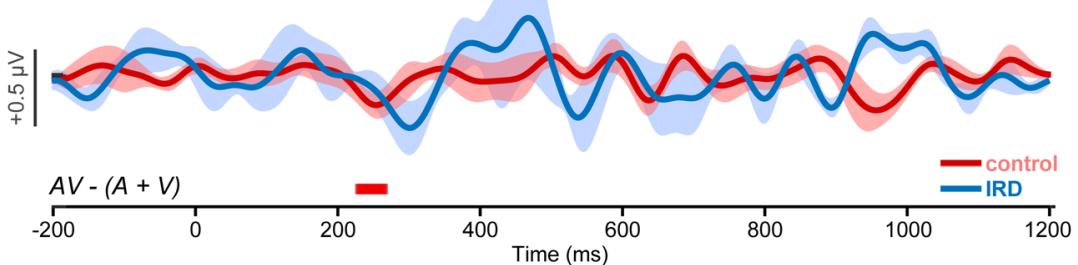

Fig. 4 Cortical event-related potentials (ERPs) following audiovisual stimuli recorded at Cz. ERPs to A, V, and AV stimuli in controls (a) and IRD (b) patients. In both groups, unimodal auditory responses elicit the largest N1/P2 deflections, with later responses for the visual N1. Across tokens, IRD patients show larger cortical responses than controls (e.g., compare N1 magnitudes). c Group comparisons of the audiovisual integration effect (difference wave) contrasting uni- and multimodal ERPs [i.e., AV - $(A+V)$ ]. Potentials different from zero indicate an enhancement in $A V$ processing compared to the summed unimodal $(A+V)$ responses. Bars below the traces show time segments where $A V>(A+V)$ (i.e., significant multisensory processing, $p<0.05)$. Despite larger unimodal responses in IRD patients, only the controls show significant AV integration ( $200 \mathrm{~ms})$ after initiation of the audiovisual stimulus. Shading $= \pm 1$ s.e.m

for a given group. We required that running significant periods persist for $>20 \mathrm{~ms}$ to be considered reliable and help further control false positives (e.g., [23]). This analysis revealed significant multimodal processing at a latency of $\sim 200 \mathrm{~ms}$ in the control cohort. The direction of this effect was negative, suggesting that the combined multimodal stimulus produced a slightly suppressed response compared to the sum of the individual constituents (i.e., coactivation). Despite the larger unimodal auditory responses (cf. Fig. 4a and b) seen in IRD patients, they did not show reliable AV enhancement. That is, IRD's multimodal response to AV stimulation was more variable and not significantly different from the summed unimodal responses, suggesting no superadditive (cf. integration) of the two senses [45].

The scalp distributions of PSD slope values $(\alpha)$ (see Fig. 2) for the A, V and AV stimuli are shown for the control group in Fig. 5a. For controls, A stimulation produced smaller PSD slope values, particularly in frontal and occipital areas, indicating more uniform EEG spectral power in these regions. Similarly, V stimuli produced smaller PSD slopes over the occipital cortex. Lastly, AV stimuli produced smaller PSD slopes primarily over frontal brain areas.
Starkly different EEG spectral power distributions were observed among IRD subjects (Fig. 5b). A and V stimulus produced an increased (steeper) EEG PSD tilt, particularly over temporal regions (e.g., auditory cortex). Paralleling controls, AV stimuli produced lower PSD slopes in the frontal and occipital areas, although the effect was weaker.

We directly compared PSD topographic maps between groups using an independent samples $t$-test corrected for multiple electrode comparisons using FDR (Fig. 5c). Results of this initial analysis (after FDR correction) showed significant group differences (i.e., IRD > control) only in the unimodal A condition for electrode clusters over the bilateral temporal and occipital cortices (channels F7, FT7, T7, F8, FT8, T8, POz, Oz; see Fig. 7, red asterisks). IRD patients had larger, more negative PSD spectral slopes than controls in these areas consistent with the ERP results (see Fig. 4).

To quantify the effects of stimulus type (A, V, AV) and group (IRD, control) on EEG spectral power slopes, we averaged PSD slopes across electrodes showing prominent group effects in our topographic analysis (e.g., Fig. 5c: F7, FT7, T7, F8, FT8, T8, POz, Oz) (Fig. 6). An ANOVA conducted on PSD values revealed a group $\mathrm{x}$ stimulus 

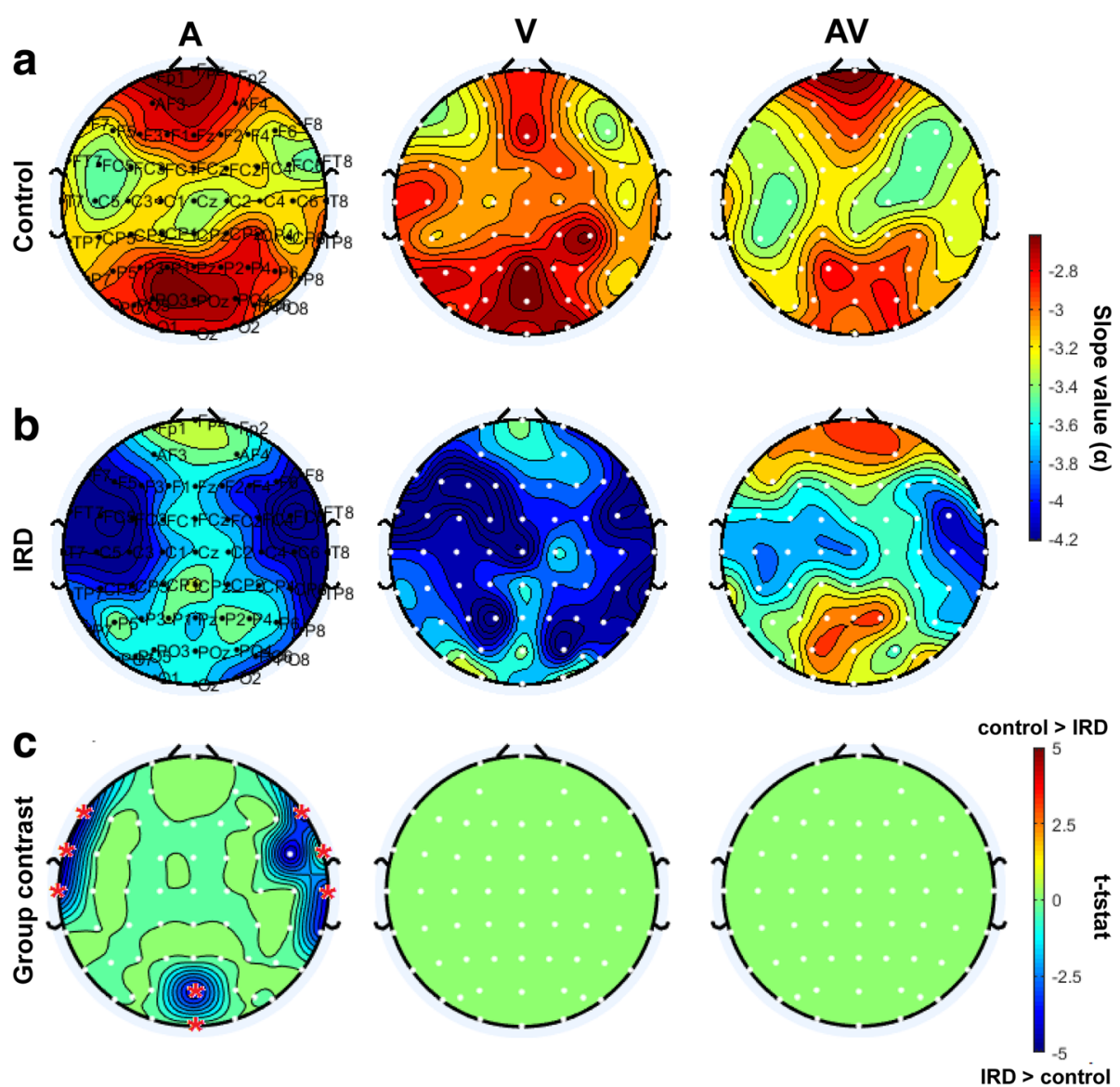

Fig. 5 Topographic maps of PSD spectral slope values (see Fig. 2) in control (a) and IRD (b) participants for A, V and AV stimuli. c Topographic map contrasting IRD and controls (i.e., Fig. 5a vs. b) (t-test; FDR threshold masked at $p<0.05)$. Significant differences in the audio-looming condition were observed between groups at temporal and occipital electrode sites ( ${ }^{*}=\mathrm{F7}$, FT7, T7, F8, FT8, T8, POz, Oz). IRD patients show increased PSD compared to controls, consistent with their larger unimodal audio responses observed in the ERPs (e.g., Fig. 4). Cool colors (i.e., negative $t$-stat) indicate scalp locations where IRD slopes > controls

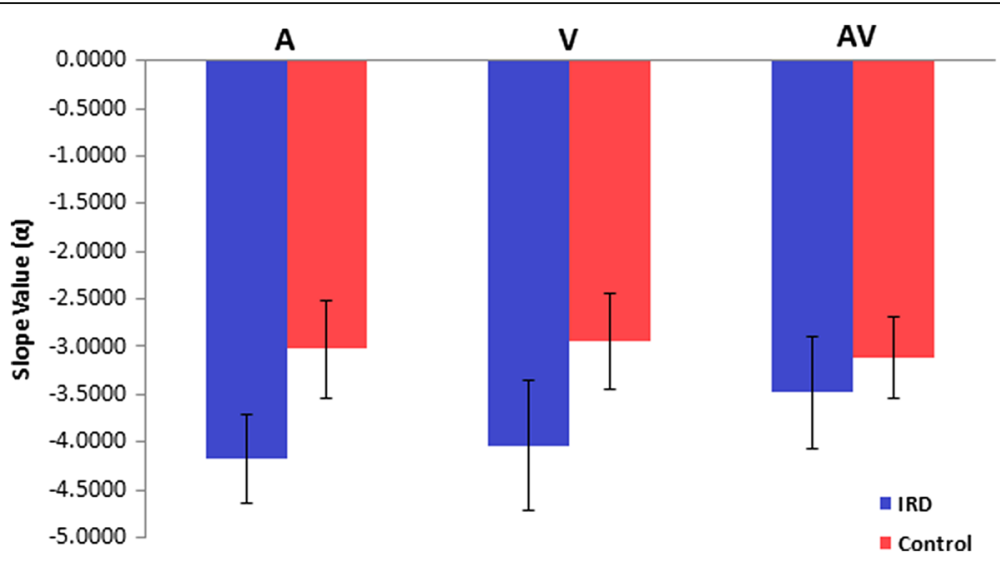

Fig. 6 Comparison of PSD slopes between IRD and controls for A, V and AV stimuli. Slope values represent the average spectral slope pooled across eight electrodes (F7, FT7, T7, F8, FT8, T8, POz, Oz). Error bars $= \pm 1$ SD 
interaction after controlling for age $\left[F_{2,33}=6.82, p=0.0033\right.$ $\left.\eta_{p}^{2}=0.29\right]$. By group, post hoc comparisons revealed that the slopes of controls did not differ across stimulus conditions (all $p$ values $>0.82$ ). In contrast, the slopes of IRD subjects were larger (i.e., more negative) for $\mathrm{V}$ compared to AV stimuli $(p=0.0008)$. Comparisons by stimulus revealed that IRD patients exhibited larger PSD slopes in the unimodal auditory (A, $p=0.029)$ and visual $(\mathrm{V}, p<0.001)$ conditions but similar responses for the multimodal AV stimulus $(p=0.65)$.

The IRD subgroups exhibited various neurological responses to the stimuli, seen in Fig. 7. RP participants appeared to have the highest average values in this group, corresponding to alpha activity across the cortex during AV stimuli. CORD participants produced very high PSD slopes in their EEG during V stimulation likely reflecting high theta-alpha activity, whereas, BBS subjects seemed to exhibit relatively normal beta-gamma activity. While these analyses suggest possible differences in AV processing between IRD pathologies, we note that the small sample size of each subgroup $(n=2)$ limits a quantitative comparison of the RP, BBS, and CORD stratifications.

Lastly, we assessed possible relations among individuals between behavioral (\% identification accuracy for "looming" stimuli, RTs) and neural (PSD) responses via Spearman correlation analyses (Fig. 8). When considering all stimuli, EEG PSD slopes were positively associated with behavioral accuracy, such that shallower (more positive) spectral slopes predicted better behavioral identification $\left(r_{s}=0.33, p=0.008\right)$ (Fig. 8a). In contrast, RTs were negatively associated with neural PSD slopes, such that that shallower (more positive) tilt of the EEG spectrum predicted faster behavioral decision times for judging AV stimuli $\left(r_{s}=-0.35, p=0.005\right)$ (Fig. 8b). Collectively, these findings help clarify the behavioral relevance of the neural PSD group effects: steeper, more negative spectral tilt of the EEG (characteristics of IRD subjects) is associated with less accurate and slower judgments of AV stimulus identity.

\section{Discussion}

In the current study, we measured cortical ERPs and spectral power of the EEG in normal controls and individuals with long-term visual impairments (IRD patients) in response to uni- and multi-modal audiovisual stimuli. Collectively, results revealed reduced behavioral sensitivity and cortical responsiveness in IRD patients (relative to controls) to stimuli when visual cues were present (i.e., $\mathrm{V}$ and AV conditions). While controls showed significant multisensory neural integration between auditory and visual inputs, we did not observe this integrative processing in IRD patients. Yet, IRDs showed larger evoked potentials than healthy controls in response to stimuli containing sound cues (A, AV tokens), an auditory bias that was paralleled in their behavioral accuracy. This latter finding implies that the auditory system may help compensate multisensory signal processing following loss or impairment of the visual sensory modality.

Notably, IRD patients show enhanced ERPs for A and AV conditions but did not show AV integration per se, as was hypothesized from non-IRD studies or other degenerative eye diseases $[29,35]$. We infer that as one sensory area (i.e., vision) declines in function over time in IRD subjects, other sensory areas (i.e., audition) are recruited to aid perceptual processing. Our findings are consistent with the notion that visual deficits due to permanent or progressive blindness are partially compensated by recruitment and/or expansion of the auditory system [17] rather than differential changes in integrative processing. Our data is also in line with studies in late-onset blindness which have shown cross modal sensory reorganization via electrophysiological

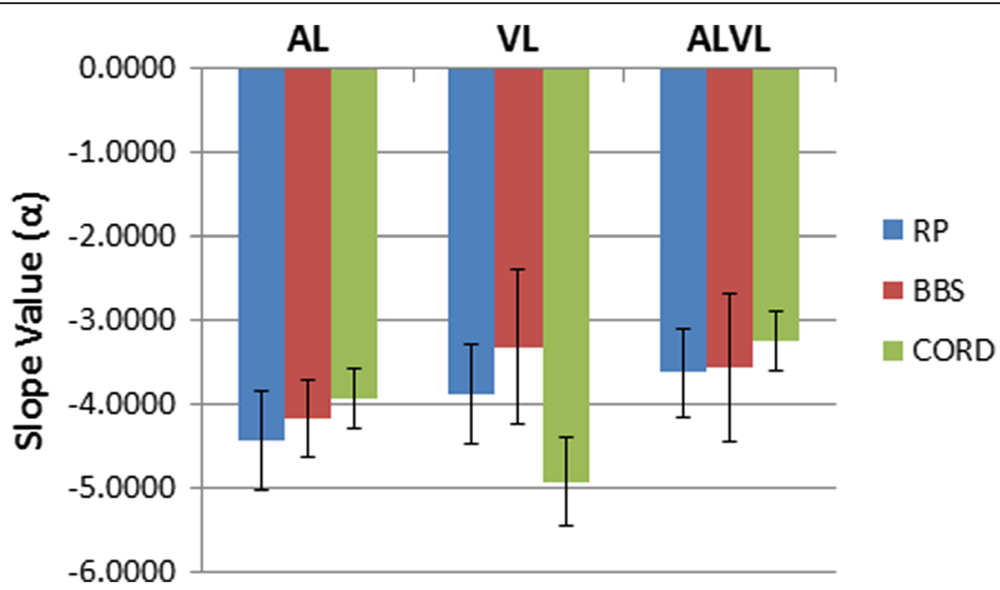

Fig. 7 AV processing in RP participants with different pathologies ( $n=2 /$ group). Bardet-Biedl syndrome (BBS) subjects exhibit similar EEG PSD slopes within a similar range, and CORD participants exhibit very high PSD slope values during $\vee$ stimulation 

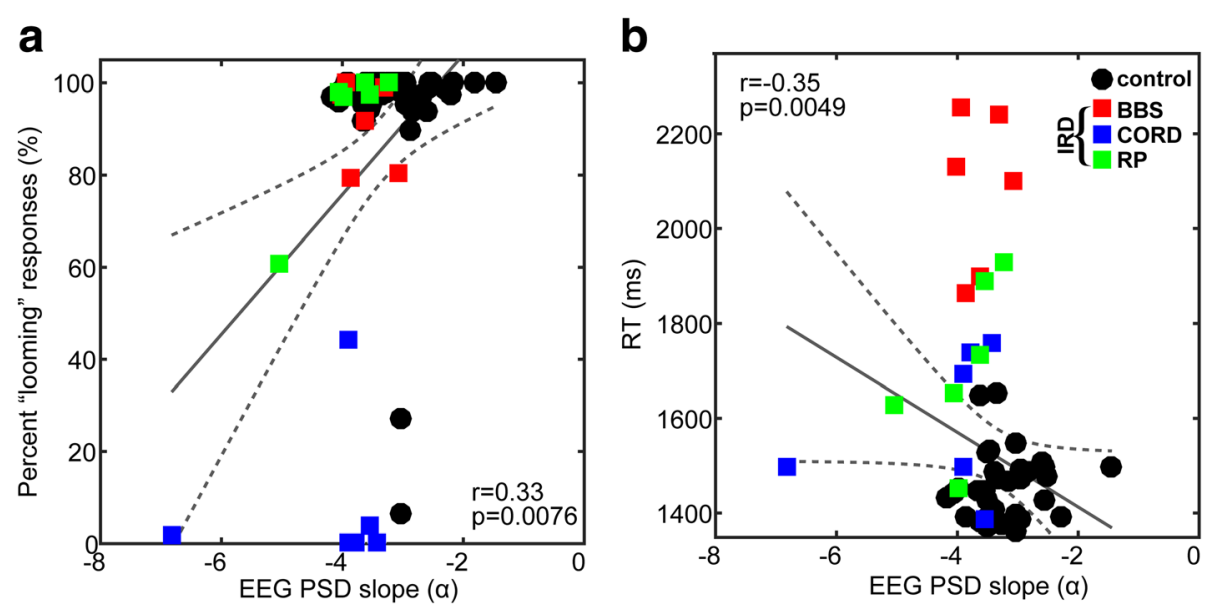

Fig. 8 Brain-behavior relations between neural PSD slopes (i.e., EEG spectral tilt) and a behavioral identification (\% "looming" responses) and b reaction times for identifying "looming" vs. "receding" motion in AV stimuli. Larger PSD slopes (i.e., less broadband EEG spectrum) indicative of IRD patients corresponds with poorer performance in identifying the perceived motion of AV stimuli. Similarly, more negative PSD slopes predict slower RTs in categorizing AV stimuli. The different etiologies of IRD (i.e., BBS, CORD, IRD) are shown as color coded symbols. Solid line, linear regression fit (MATLAB fitlm, bisquare weighting) to the pooled data (across all subjects); Dotted lines, 95\% Cl

recordings [39]. Visual cortical reorganization may involve normally-developed striate and extrastriate visual areas, which are presumably involved in visual imagery [40]. Late-onset blindness utilizes these formally developed cortical structures which may be activated through bidirectional auditory and visual sensory pathways. The occipital cortex can be activated through non-visual stimulus in blind subjects via auditory tonal stimuli, suggesting that primary visual areas may be not be as sensory specific as traditionally thought [40]. It is conceivable that, in cases of progressively degenerating vision (as in IRDs), the brain initiates sensory reorganization and compensates by recruiting multisensory neurons in both auditory and visual cortices. This may account for the larger responsiveness to stimuli containing auditory cues in IRD subjects relative to controls.

In this regard, our data are in agreement with fMRI studies which reveal an expansion of the tonotopic maps of auditory cortex in blind patients compared to sighted individuals [17]. These studies suggest that visually impaired individuals tend to exhibit greater reliance on other sensory inputs to maintain the same degree of perceptual discrimination. Our results demonstrate a similar entrainment of large cortical populations from auditory stimulation in IRD subjects. Due to the gradual loss of vision in IRDs, larger neural networks might be constructed via activation of previously dormant connections and/or emergence of new neural pathways between distant cortical regions.

In particular, our behavioral identification results (Fig. 3a) are consistent with the fact that, in IRD subjects like the ones included in this investigation without syndromic hearing impairment (e.g., Usher syndrome), the sensory deficit is purely visual in nature, thus compromising behavioral identification of AV stimuli. In today's society, our interaction with the environment and others have become far more visual than auditory (e.g., most people age 45 or below nowadays communicate via text and social media than by phone call). Thus, the fact that the behavioral identification of auditory (A) tokens by IRD patients was not proportionally lower as seen in controls suggests that, in controls, visual cues are cognitively the ones relied upon the most, whereas IRD subjects, being visually impaired, compensate proportionally with higher sensitization to their auditory function (e.g., Fig. 4b). Nevertheless, the slower RTs for IRD subjects in these conditions suggest this enhanced auditory sensitivity is at the expense of slower processing, presumably reflecting a compensatory strategy from the impaired visual input.

Analysis of EEG frequency power revealed increased spectral slopes (Fig. 5b) in the IRD group relative to controls for simultaneous A and V stimulation and singular $\mathrm{V}$ stimulation. Increase in EEG slope could reflect higher lower frequency energy in A and decreased higher frequency energy for $\mathrm{V}$, consistent with the interpretation of low and high bands as network communication and stimulus coding, respectively. In brief, we found that larger (more negative) slopes are associated with poorer AV perception (Fig. 5c). If auditory responses are dominated by increased alpha activity in IRDs relative to controls, this effect may point to an enhancement in auditory sensitivity of IRD subjects, as suggested by the steeper EEG spectral slopes (Fig. 5b) and larger ERPs as we find in 
auditory-only conditions (Fig. 4). Interestingly, spectral and temporal enhancements in IRD's brain responses were observed in the absence of AV integration (Fig. 4c). Thus, an alternate account of the observed IRD auditory enhancements may be that long-term visual deprivation causes higher-level cortical disinhibition that produces broad activation and permits enhanced neural encoding of sound. Indeed, complementary studies have suggested that other forms of sensory loss/deprivation (e.g., hearing loss) can alter cortical response activity due to a disinhibition of sensory coding ([13]b).

Our study aimed to identify changes in cortical processing of multimodal stimuli following long term visual loss. For IRD subjects, we observed that auditory inputs engage more responsiveness from the cortex and seem to entrain neural activity at lower frequency bands of the EEG, particularly at scalp locations over temporal and parietal-occipital junctions. Larger responsivity in these areas could reflect changes in neural resources mediating task execution. For example, it is conceivable that given their partial sensory deficit, IRD individuals required higher levels of attentional deployment or cognitive processing to arrive at their behavioral judgments. This notion is supported by our behavioral RT data, which showed much longer response times in IRDs relative to controls when identifying the perceived motion of AV stimuli (Fig. 3b). As stated by Smilek et al. [44] and Myles et al. [35], higher-level brain regions may collect information transmitted from various sensory cortices, and project this information to the brain areas eliciting the concurrent perception. It is conceivable this information routing is still possible but simply more sluggish in IRD. This proposition is evident by the chance behavioral accuracy at the expense of slower response times exhibited by IRD subjects (Fig. 3).

Several frameworks have been developed to describe how brain networks manage simultaneous sensory inputs. The disinhibited feedback model suggests that multi-sensory input results in disinhibited feedback from higher-level cortical areas in the processing hierarchy [1, 22]. This would imply that, for AV stimulation, higherlevel cortical areas collect information transmitted from the sensory cortex, and project this information to the brain areas eliciting the concurrent percept. Previous studies have demonstrated that areas of the posterior inferior temporal cortex, the parieto-occipital junction, and V4 were activated during word listening more than during tone listening in individuals with congenital cross-sensory integration deficits [35, 44]. In this regard, a specific sensory deficit in one modality may induce a form of cortical remapping and recruit compensatory processing in brain areas not associated with the impairment (e.g., recruitment of auditory cortex in blind individuals; [17]).
Multisensory processing can be directly assessed using audiovisual AV looming and receding stimuli, which convey the sense of motion [14]. For these stimuli, the integration of $\mathrm{A}$ and $\mathrm{V}$ looming signals may be mediated by functional interactions between primary auditory cortex and the superior temporal sulcus (STS), two areas involved in integrating behaviorally relevant AV signals $[3,19,31]$. AV looming signals also elicit increased gamma-band coherence between these areas, relative to unimodal (A or V stimulation) or receding motion signals [31]. Increased neuronal coherence might result in more efficient communication between these areas and fronto-parietal networks [21, 42, 47], resulting in bettercoordinated responses to looming (i.e., approaching) events [2, 43]. The STS is known to be involved in the perception of biological motion, which may explain why impending signals seem to heavily recruit this area and reveal the largest group differences in our neural data (Fig. 5c). Germane to the current study, AV processing is not static, but has been shown to vary with certain experiential factors (e.g., musical training) and learning $[8,38]$. While AV processing can be positively enhanced with learning and experience presumably, we show here that it can be negatively altered in cases of visual sensory deficit.

Limitations of this study are worth noting. While group differences in the EEG between IRDs and controls (pooled across conditions in Fig. 6) showed a large effect size $(d=2.07 ; \alpha=0.05$, power $=98 \%$, twotailed $t$-test), our limited sample of different IRD subgroups (i.e., Fig. 7) limits our conclusions regarding possible differential effects in AV processing between $\mathrm{RP}, \mathrm{BBS}$, and CORD pathologies. Indeed, comparisons between the RP and CORD spectral slopes in the V condition (which shows the largest subgroup differences in Fig. 7) achieves an effect size of $d=0.81$. However, this result is tempered by the fact that the corresponding power is only $7 \%$. [n=50 subjects would be needed to detect an effect at $80 \%$ power]. Additional studies on larger population samples, especially by disease subgroup, are needed to confirm the different trends noted here in handling looming vs. receding stimuli depending on the vision loss being peripheral (RP) vs. central (CORD), and in subjects with Bardet-Biedl syndrome (BBS). It is possible that comparing different stratifications of IRD may prove difficult given heterogeneity between the populations. Nevertheless, we demonstrate that EEG responses can reveal robust differences between IRD patients and normal controls. We anticipate that the larger study population planned for in this study would not only reinforce our initial findings, but clearly delineate which subtype produced the highest degree of auditory compensation. 


\section{Conclusion}

Neural activity in IRD subjects offer insight into how auditory and visual sensory inputs are processed when the visual input is diminished over time. We found evidence for enhanced (or at least more sensitized) auditory neural encoding in participants with IRDs, which may relate to compensatory recruitment of auditory system function with gradual visual loss and increased sensitization, or differential reweighting of the senses, towards sound processing. Our approach utilized salient multisensory stimuli that revealed compensatory neuroplasticity in the brain processing of IRD patients. More broadly, this approach could be applied in a rehabilitation/training paradigm to further enhance auditory sensitivity in cases of progressive visual loss in attempts to enhance function in one sensory area as the input to another modality diminishes.

\section{Additional file}

Additional file 1: Figure S1. IRD patient selection criteria. (DOCX $761 \mathrm{~kb}$ )

\section{Abbreviations}

A + V: Summed unimodal; A: Auditory; AV: Audiovisual; BBS: Bardet-biedl syndrome; CORD: Cone-rod dystrophy; EEG: Electroencephalography; ERG: Electroretinogram; ERP: Event-related brain potentials; FAF: Fundus Autofluorescence; fMRI: Functional magnetic resonance imagery; IRDs: Inherited retinal dystrophies; PCA: Principal component analysis; PSD: Power spectral density; RP: Retinitis pigmentosa; RT: Reaction times; SD-OCT: Spectral domain optical coherence tomography; STS: Superior temporal sulcus; V: Visual

\section{Acknowledgements}

We would also like to gratefully acknowledge the research coordinating assistance of Dr. Barbara J. Jennings.

\section{Funding}

This investigation was supported initially by an unrestricted grant from Research to Prevent Blindness, Inc., New York, NY to the University of Tennessee Health Science Center Department of Ophthalmology, Memphis, TN, and, presently, by an identical RPB grant to the Duke Eye Center, Duke University School of Medicine, Durham, NC.

\section{Availability of data and materials}

The datasets used and/or analyzed during the current study are available from the corresponding author on reasonable request.

\section{Authors' contributions}

Al examined and selected patients for this study. MHM and GMB analyzed the data. MHM, Al, GMB interpreted results and wrote and approved the final manuscript.

\section{Ethics approval and consent to participate}

Each participant gave informed written consent in compliance with a joint protocol approved by the Institutional Review Boards of the University of Tennessee Health Science Center (IRB \#: 13-02782-XP) and the University of Memphis (IRB \#2370).

\section{Consent for publication}

Not applicable

\section{Competing interests}

The authors declare that they have no competing interests.

\section{Publisher's Note}

Springer Nature remains neutral with regard to jurisdictional claims in published maps and institutional affiliations.

\section{Author details}

${ }^{1}$ Department of Anatomy and Neurobiology, University of Tennessee Health Sciences Center, Memphis, TN 38163, USA. '2Department of Ophthalmology, Center for Retinal Degenerations and Ophthalmic Genetic Diseases, Duke University School of Medicine, Durham, NC, USA. ${ }^{3}$ School of Communication Sciences \& Disorders, University of Memphis, Memphis, TN, USA. ${ }^{4}$ Institute for Intelligent Systems, University of Memphis, Memphis, TN, USA.

Received: 5 July 2017 Accepted: 29 November 2017

Published online: 07 December 2017

\section{References}

1. Armel KC, Ramachandran VS. Acquired Synesthesia in retinitis Pigmentosa. Neurocase. 1999; doi:10.1080/13554799908411982.

2. Bach DR, Schachinger H, Neuhoff JG, Esposito F, Di Salle F, Lehmann C, Seifritz E. Rising sound intensity: an intrinsic warning cue activating the amygdala. Cereb Cortex. 2008; doi:10.1093/cercor/bhm040.

3. Barraclough NE, Xiao D, Baker Cl, Oram MW, Perrett DI. Integration of visual and auditory information by superior temporal sulcus neurons responsive to the sight of actions. J Cogn Neurosci. 2005; doi:10.1162/0898929053279586.

4. Benjamini $Y$, Hochberg $Y$. Controlling the false discovery rate: a practical and powerful approach to multiple testing. J R Stat Soc Ser B Methodol. 1995; doi:10.2307/2346101

5. Benoit MM, Raij T, Lin FH, Jaaskelainen IP, Stufflebeam S. Primary and multisensory cortical activity is correlated with audiovisual percepts. Hum Brain Mapp. 2010; doi:10.1002/hbm.20884.

6. Besle J, Fischer C, Bidet-Caulet A, Lecaignard F, Bertrand O, Giard MH. Visual activation and audiovisual interactions in the auditory cortex during speech perception: intracranial recordings in humans. J Neurosci. 2008; doi:10.1523/JNEUROSCI.2875-08.2008

7. Bidelman GM. Induced neural beta oscillations predict categorical speech perception abilities. Brain Lang. 2015; doi:10.1016/j.bandl.2014.11.003.

8. Bidelman GM. Musicians have enhanced audiovisual multisensory binding: experience-dependent effects in the double-flash illusion. Exp Brain Res. 2016; doi:10.1007/s00221-016-4705-6.

9. Bidelman GM. Amplified induced neural oscillatory activity predicts musicians' benefits in categorical speech perception. Neuroscience. 2017: doi:10.1016/j.neuroscience.2017.02.015.

10. Bidelman GM, Chung WL. Tone-language speakers show hemispheric specialization and differential cortical processing of contour and interval cues for pitch. Neuroscience. 2015; doi:10.1016/j.neuroscience.2015.08.010.

11. Bidelman GM, Grall J. Functional organization for musical consonance and tonal pitch hierarchy in human auditory cortex. Neurolmage. 2014; doi:10.1016/j.neuroimage.2014.07.005

12. Bidelman GM, Moreno S, Alain C. Tracing the emergence of categorical speech perception in the human auditory system. Neurolmage. 2013; doi:10.1016/j.neuroimage.2013.04.093

13. Bidelman GM, Villafuerte JW, Moreno S, Alain C. Age-related changes in the subcortical-cortical encoding and categorical perception of speech. Neurobiol Aging. 2014; doi:10.1016/j.neurobiolaging.2014.05.006.

14. Cappe C, Thelen A, Romei V, Thut G, Murray MM. Looming signals reveal synergistic principles of multisensory integration. J Neurosci. 2012; doi:10.1523/JNEUROSCI.5517-11.2012

15. Cox KF, Kerr NC, Kedrov M, Nishimura D, Jennings BJ, Stone EM, lannaccone A. Phenotypic expression of Bardet-Biedl syndrome in patients homozygous for the common M390R mutation in the BBS1 gene. Vis Res. 2012; doi:10.1016/j.visres.2012.08.005.

16. Delorme A, Makeig S. EEGLAB: an open source toolbox for analysis of single-trial EEG dynamics including independent component analysis. J Neurosci Methods. 2004;134(1):9-21.

17. Elbert T, Sterr A, Rockstroh B, Pantev C, Muller MM, Taub E. Expansion of the tonotopic area in the auditory cortex of the blind. J Neurosci. 2002;22(22): 9941-4.

18. Fister J, Stevenson RA, Nidiffer AR, Barnett ZP, Wallace MT. Stimulus intensity modulates multisensory temporal processing. Neuropsychologia. 2016; doi:10.1016/j.neuropsychologia.2016.02.016. 
19. Ghazanfar AA, Maier JX, Hoffman KL, Logothetis NK. Multisensory integration of dynamic faces and voices in rhesus monkey auditory cortex. J Neurosci. 2005; 25(20):5004-12.

20. Gougoux F, Zatorre R, Lassonde M, Voss P, Lepore F. A functional neuroimaging study of sound localization: visual cortex activity predicts performance in earlyblind individuals. PLoS Biol. 2005; doi:10.1371/journal.pbio.0030027.

21. Graziano MS, Cooke DF. Parieto-frontal interactions, personal space, and defensive behavior. Neuropsychologia. 2006;44(13):2621-35.

22. Grossenbacher PG, Lovelace CT. Mechanisms of synesthesia: cognitive and physiological constraints. Trends Cogn Sci. 2001;5(1):36-41.

23. Guthrie D, Buchwald JS. Significance testing of difference potentials. Psychophysiology. 1991;28(2):240-4.

24. Hall DA, Fussell C, Summerfield AQ. Reading fluent speech from talking faces: typical brain networks and individual differences. J Cogn Neurosci. 2005:17(6):939-53.

25. lannaccone A. Genotype-phenotype correlations and differential diagnosis in autosomal dominant macular disease. Doc Ophthalmol. 2001;102:197-236.

26. lannaccone $\mathrm{A}$. The genetics of hereditary retinopathies and optic neuropathies. Comp Ophthalmol Update. 2005;5:39-62.

27. Iannaccone A, De Propris G, Roncati S, Rispoli E, Del Porto G, Pannarale MR. The ocular phenotype of the Bardet-Biedl syndrome. Comparison to nonsyndromic retinitis pigmentosa. Ophthalmic Genet. 1997;18:13-26.

28. lannaccone A, Mykytyn K, Persico AM, Searby CC, Baldi A, Jablonski MM, Sheffield VC. Clinical evidence of decreased olfaction in Bardet-Biedl syndrome caused by a deletion in the BBS4 gene. Am J Med Genet A. 2005; doi:10.1002/ajmg.a.30512.

29. Jancke L, Rogenmoser L, Meyer M, Elmer S. Pre-attentive modulation of brain responses to tones in coloured-hearing synesthetes. BMC Neurosci. 2012; doi:10.1186/1471-2202-13-151.

30. Kriegstein K. The neural bases of multisensory processes. Boca Raton (FL): CRC press/Taylor \& Francis;" Murray MM, Wallace MT, editors, Chapter 34A. 2012.

31. Maier JX, Chandrasekaran C, Ghazanfar AA. Integration of bimodal looming signals through neuronal coherence in the temporal lobe. Curr Biol. 2008; doi:10.1016/j.cub.2008.05.043.

32. Maier JX, Neuhoff JG, Logothetis NK, Ghazanfar AA. Multisensory integration of looming signals by rhesus monkeys. Neuron. 2004;43(2):177-81.

33. Myers MH, Kozma R. Seizure prediction through dynamic synchronization measures of neural populations. Paper presented at the 2009 International Joint Conference on Neural Networks; 2009. doi:10.1109/IJCNN.2009.5179083.

34. Myers MH, Kozma R. Modeling normal/epileptic brain dynamics with potential application in titration therapy. Paper presented at the neural networks (IJCNN), the 2011 international joint conference on; 2011. doi:10.1109/IJCNN.2011.6033629.

35. Myles KM, Dixon MJ, Smilek D, Merikle PM. Seeing double: the role of meaning in alphanumeric-colour synaesthesia. Brain Cogn. 2003; doi:10.1016/S0278-2626(03)00139-8

36. Neuhoff JG. Perceptual bias for rising tones. Nature. 1998; doi:10.1038/25862

37. Pekkola J, Ojanen V, Autti T, Jaaskelainen IP, Mottonen R, Tarkiainen A, Sams M. Primary auditory cortex activation by visual speech: an fMRI study at $3 \mathrm{~T}$. Neuroreport. 2005;16(2):125-8.

38. Powers AR 3rd, Hillock AR, Wallace MT. Perceptual training narrows the temporal window of multisensory binding. J Neurosci. 2009; doi:10.1523/JNEUROSCI.3501-09.2009.

39. Rao A, Nobre AC, Alexander I, Cowey A. Auditory evoked visual awareness following sudden ocular blindness: an EEG and TMS investigation. Exp Brain Res. 2007;176(2):288-98.

40. Roder B, Rosler F, Neville HJ. Auditory memory in congenitally blind adults: a behavioral-electrophysiological investigation. Brain Res Cogn Brain Res. 2001; doi:10.1016/S0926-6410(01)00002-7

41. Romei V, Murray MM, Cappe C, Thut G. Preperceptual and stimulus-selective enhancement of low-level human visual cortex excitability by sounds. Curr Biol. 2009; doi:10.1016/j.cub.2009.09.027

42. Saalmann YB, Pigarev IN, Vidyasagar TR. Neural mechanisms of visual attention: how top-down feedback highlights relevant locations. Science. 2007; doi:10.1126/science.1139140.

43. Seifritz E, Neuhoff JG, Bilecen D, Scheffler K, Mustovic H, Schachinger H, et al. Neural processing of auditory looming in the human brain. Curr Biol. 2002; doi:10.1016/S0960-9822(02)01356-8.

44. Smilek D, Dixon MJ, Cudahy C, Merikle PM. Synaesthetic photisms influence visual perception. J Cogn Neurosci. 2001; doi:10.1162/089892901753165845.
45. Tyll S, Bonath B, Schoenfeld MA, Heinze H-J, Ohl FW, Noesselt T. Neural basis of multisensory looming signals. Neurolmage, 2013; doi.org/10.1016/j. neuroimage.2012.09.056.

46. Wallstrom GL, Kass RE, Miller A, Cohn JF, Fox NA. Automatic correction of ocular artifacts in the EEG: a comparison of regression-based and componentbased methods. Int J Psychophysiol. 2004; doi:10.1016/j.jpsycho.2004.03.007.

47. Womelsdorf T, Schoffelen JM, Oostenveld R, Singer W, Desimone R, Engel AK, Fries P. Modulation of neuronal interactions through neuronal synchronization. Science. 2007; doi:10.1126/science.1139597.

\section{Submit your next manuscript to BioMed Central and we will help you at every step:}

- We accept pre-submission inquiries

- Our selector tool helps you to find the most relevant journal

- We provide round the clock customer support

- Convenient online submission

- Thorough peer review

- Inclusion in PubMed and all major indexing services

- Maximum visibility for your research

Submit your manuscript at www.biomedcentral.com/submit
Biomed Central 